\title{
Dwarf Archives: A Compendium of M, L, and T Dwarf Data
}

\author{
Christopher R. Gelino*, J. Davy Kirkpatrick ${ }^{\dagger}$ and Adam J. Burgasser** \\ * Spitzer Science Center, California Institute of Technology, 1200 E. California Blvd. Pasadena, CA \\ 91125 \\ ${ }^{\dagger}$ Infrared Processing and Analysis Center, California Institute of Technology, 1200 E. California \\ Blvd. Pasadena, CA 91125 \\ ** Kavli Institute for Astrophysics \& Space Research, Massachusetts Institute of Technology, \\ Cambridge, MA 02139
}

\begin{abstract}
Dwarf Archives is an online archive containing astrometric, photometric, and spectroscopic information for all known L and T dwarfs (over 650 objects), as well as a select sample of over $500 \mathrm{M}$ dwarfs. In this paper, we describe the specific data available in the archive, the interface for searching through it and discuss plans for future updates.
\end{abstract}

Keywords: Catalogs; Stars: low-mass, brown dwarfs; Stars: late-type

PACS: 95.80.+p; 97.20.Jg; 97.20.Vs

\section{INTRODUCTION}

The number of L and T dwarfs has been growing steadily since their first discovery over 10 years ago. Currently, there are over 600 objects known with these spectral types. While SIMBAD is an excellent resource for finding information about stars from wellstudied catalogs, the data present for L and T dwarf objects are spotty. Dwarf Archives (see http: / / DwarfArchives.org) was created to provide a single online source in which to find basic information about all $\mathrm{L}$ and $\mathrm{T}$ dwarfs. It is largely based on the M, L, and T dwarf archive described in Kirkpatrick (2003), with additional data and concepts from the T Dwarf database (compiled by AJB) and a brown dwarf database (created by CRG). Dwarf Archives was originally launched in January 2005 and has grown in both the number of entries and the complexity of the interface since then. It is in use by hundreds of astronomers throughout the world and maintains a steady usage.

While the L \& T dwarf database remains the main driver for Dwarf Archives, the archive now includes spectra for over 500 of the nearest and brightest $\mathrm{M}$ dwarfs. With this data release, we expect to attract a larger number of users who visit the site just for these spectra. In this article we briefly describe the contents of the archive and how it might be used to facilitate studies of M, L, and T dwarfs.

\section{DWARF ARCHIVES CONTENTS}

As alluded to above, the data present in Dwarf Archives are divided into two separate databases: the $\mathrm{L} \& \mathrm{~T}$ dwarf database and the $\mathrm{M}$ dwarf database. The $\mathrm{L} \& \mathrm{~T}$ dwarf database is intended to be a complete listing of all spectroscopically classified L \& T 
TABLE 1. Data contents of Dwarf Archives

\begin{tabular}{lr}
\hline Data & Database \\
\hline 2MASS photometry, astrometry, \& designation & both \\
discovery name and ref. & L \& T dwarfs \\
proper motion and ref. & L \& T dwarfs \\
trigonometric parallax and ref. & L \& T dwarfs \\
optical spectral type and ref. & both \\
near-IR spectral type and ref. & L \& T dwarfs \\
special notes (eg. binarity) & L \& T dwarfs \\
Gl/GJ name & M dwarf \\
LHS name & M dwarf \\
other common name & M dwarf \\
optical spectra & M dwarf \\
\hline
\end{tabular}

dwarfs, whereas the $\mathrm{M}$ dwarf archive is a collection of homogeneously typed, bright and nearby stars. Therefore, the two databases have different uses and intended users. Nonetheless, many data in Dwarf Archives are common to both databases. Table 1 details the currently available data and in which database the data can be found. In the next two sections, we describe the data that are unique to each database.

\section{L and T Dwarf Database}

Dwarf Archives is the only online database containing photometry and astrometry for all known spectroscopically classified L and T dwarfs. As of October 2008 there are over 650 objects in our database with either an optical or a near-IR spectral type of L or T. These objects are spread over the entire sky (Fig. 1), but are more concentrated in the northern hemisphere. This dichotomy is likely caused by the northern-hemisphere based SDSS survey for which $149 \mathrm{~L}$ and T dwarfs have been discovered above the ecliptic plane versus only 36 below the ecliptic. Table 2 provides the full details for our $\mathrm{L}$ and $\mathrm{T}$ dwarf database. In order to homogenize the database as much as possible, the photometry is largely from the 2MASS Point Source Catalog (PSC). We supplement the 2MASS photometry with other published near-IR photometry for objects that are not in the 2MASS PSC (these objects are either too faint or too close to a bright star). More than half of the catalog has measured proper motions and 81 have trigonometric parallax measurements 1 . By requirement, all of these objects have either an optical or near-IR spectral type (many have both). Finally, we include notes about peculiar objects (eg. binaries and low gravity objects).

\footnotetext{
${ }^{1}$ We do not include photometric parallaxes because that quantity is not a direct measurement.
} 
TABLE 2. Summary of information in the databases

\section{L \& T Dwarf database}

\begin{tabular}{lc}
\hline Total objects & $668^{*}$ \\
\hline L Dwarfs (optical or near-IR types) & 528 \\
T Dwarfs (optical or near-IR types) & 146 \\
optical spectral types & 428 \\
near-IR spectral types & 342 \\
proper motions & 330 \\
parallaxes & 81 \\
\hline
\end{tabular}

\section{Dwarf database}

\begin{tabular}{lr}
\hline Total objects & 536 \\
\hline M dwarfs/giants & 507 \\
K dwarfs/giants & 27 \\
dwarf carbons & 2 \\
optical spectra & 682 \\
\hline
\end{tabular}

* Note that the sum of the $\mathrm{L}$ and $\mathrm{T}$ dwarfs does not equal the total number of objects in the database. This is because several objects have very different spectral types in the optical and the near-IR. For example, SDSS 042348.57-041403.5 has an optical spectral type of L7.5 (Cruz et al. 2003) and a near-IR type of T0 (Burgasser et al. 2006). Thus, it is included in the totals for the L dwarfs and the T dwarfs.

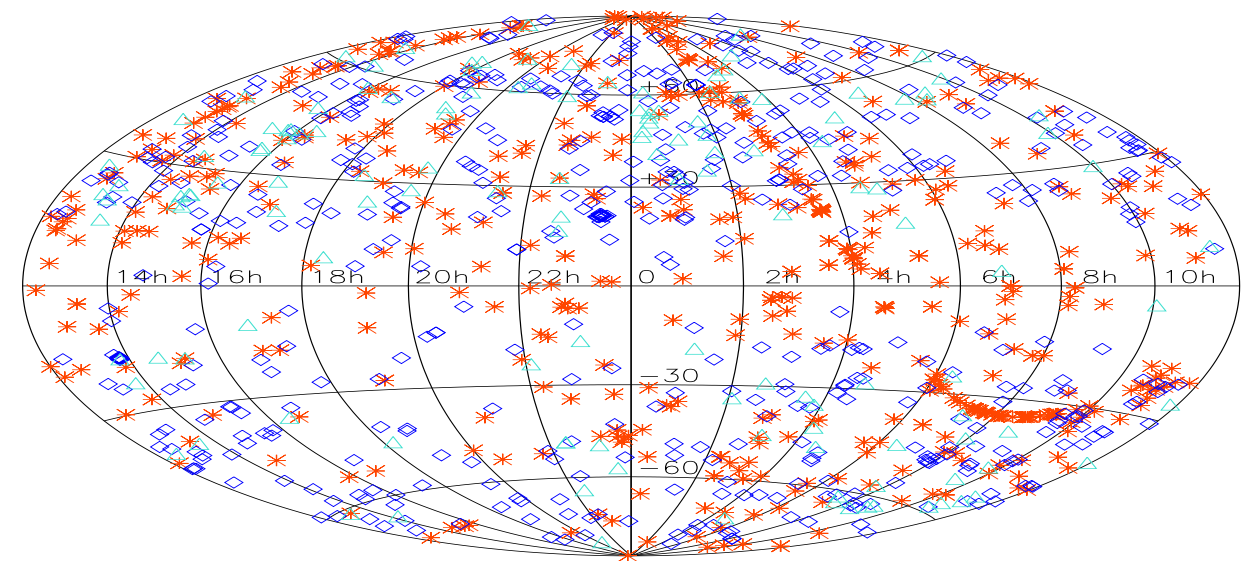

FIGURE 1. This figure shows the all-sky distribution of objects in these databases. Orange-red asterisks are objects in the $\mathrm{M}$ dwarf database, blue diamonds are $\mathrm{L}$ dwarfs, and turquoise triangles are $\mathrm{T}$ dwarfs.

\section{Dwarf Database}

The $M$ dwarf database contains a collection of $\approx 680$ optical spectra for many nearby, bright $M$ dwarfs and various other late-type stars (Table 2) spread throughout the sky (Fig. 1). As with the L \& T dwarf database, we have 2MASS J, H, \& Ks photometry and astrometry for nearly all objects. Whereas the spectral types for the L and T dwarfs have been performed by many different astronomers with sometimes subtly different methods, the $\mathrm{M}$ dwarf spectral types were homogeneously classified by one person (JDK), allowing for much more meaningful comparisons among spectral type trends. 


\section{SEARCHES AND RESULTS}

Search forms are available for both databases that allow for a large number of parameters to be simultaneously constrained. All of the numerical fields (i.e. photometry and astrometry) and the spectral types can be used in parameter searches. Searches around a sky position or a specific object (object names are resolved with SIMBAD and NED using the IRSA LookUp service) can also be performed. For example, it is possible to search for objects within a specific range of RA, that are brighter than a given magnitude, and that fall within a certain color range. The user can also choose which fields are to be returned and how those results are to be ordered. Hyperlinks to the ADS Abstract Service are provided for all references. Also, multi-wavelength finder charts can be obtained through links to the IRSA Finder Chart service. Finally, results from M dwarf queries requesting optical spectra have links to quick-look plots of the spectra as well as options for downloading ASCII versions of the spectra.

\section{FUTURE PLANS}

The information in Dwarf Archives continues to be updated as new objects are discovered and new data about existing objects are measured. Because many of the new L \& T dwarf discoveries are from very deep surveys (UKIDSS, SDSS), an increasing number of L \& T dwarfs are not in the 2MASS PSC. In an effort to clarify 2MASS photometry from non-2MASS photometry, we are planning on separating 2MASS PSC photometry from photometry from other systems. Our goal is to supplement the 2MASS photometry with photometry from SDSS $(r, i, \& z)$, UKIDSS (Y, J, H, \& K), MKO system measurements (z, Y, J, H, K, Ks, L', \& M'), DENIS (I, J, \& K), and Spitzer IRAC (Channels $1,2,3, \& 4)$. We also have plans to incorporate optical and near-IR spectra (including previews) for L and T dwarfs. Finally, we have a large set of optical spectra of M dwarfs from Keck/LRIS that would beneficial to the M dwarf database.

\section{ACKNOWLEDGMENTS}

The success of this archive depends immensely on support from the community. We appreciate all past, present, and future assistance that you may provide. This work makes use of the 2MASS archive, the ADS abstract service, and several IRSA services. Our server was funded by a NASA Small Research Grant administered by the American Astronomical Society. We thank them for their generosity.

\section{REFERENCES}

1. A. J. Burgasser et al. ApJ 637, 1067-1093 (2006).

2. K. L. Cruz et al. AJ 126, 2421-2448 (2003).

3. J. D. Kirkpatrick, "2MASS Data Mining and the M, L, and T Dwarf Archives," in Brown Dwarfs, edited by E. L. Martín, IAU Symposium 211, ASP, San Francisco, 2003, p. 189. 